\title{
Vertebral Lateral Notch as Optimal Entry Point for Lateral Mass Screwing Using Modified Roy-Camille Technique
}

\author{
Norio Yamamoto, Hirofumi Kosaka, Kosaku Higashino, Masatoshi Morimoto, Kazuta Yamashita, \\ Fumitake Tezuka, Fumio Hayashi, Yoichiro Takata, Toshinori Sakai, Akihiro Nagamachi, Koichi Sairyo \\ Department of Orthopedics, Institute of Biomedical Sciences, University of Tokushima Graduate School, Tokushima, Japan
}

Study Design: Retrospective study of 37 consecutive female patients with cervical spondylotic myelopathy who underwent reconstructed computed tomography (CT) scanning of the cervical spine.

Purpose: The purpose of this study was to investigate whether the vertebral lateral notch of the cervical spine is an effective landmark to determine the entry point for lateral mass screwing. A modified Roy-Camille technique was used to determine the entry point associated with the lateral notch of the cervical spine.

Overview of Literature: The Roy-Camille technique has been a popular technique for the posterior fixation of the cervical spine. A problem with this technique is determining the entry point on the lateral mass via visual inspection, such as in cases with degenerative or destructive cervical facet joints.

Methods: Thirty-three female patients with cervical spondylotic myelopathy underwent reconstructed CT scanning of the cervical spine. Overall, 132 vertebrae from C3 to C6 were reviewed using reconstructed CT. The probable trajectory using a modified RoyCamille technique was determined using reconstructed CT scans, and the optimal entry point was identified. Horizontal and vertical distances from the vertebral lateral notch were measured.

Results: The entry point determined using the modified Roy-Camille technique was significantly superior and medial compared with that determined using the conventional Roy-Camille technique. At C3 and C4 levels, the entry point using the modified technique was $1.4 \mathrm{~mm}$ below and $4.4 \mathrm{~mm}$ medial to the lateral notch, and at C5 and C6 levels, it was $2.3 \mathrm{~mm}$ below and $4.9 \mathrm{~mm}$ medial to the lateral notch.

Conclusions: The vertebral lateral notch of the cervical spine was an effective landmark to determine the entry point for lateral mass screwing. The modified Roy-Camille technique proposed here may prevent surgical complications and poor outcomes.

Keywords: Lateral vertebral notch; Roy-Camille technique; Lateral mass screwing

\section{Introduction}

Lateral mass screwing was first reported by Roy-Camille et al. [1] in 1972, and several reports have described technical variations to improve the mechanical competence and anatomic safety of the technique. The Roy-Camille

Received Feb 27, 2017; Revised May 31, 2017; Accepted Jun 21, 2017

Corresponding author: Kosaku Higashino

Department of Orthopedics, Institute of Biomedical Sciences, University of Tokushima Graduate School, 3-18-15 Kuramoto, Tokushima 770-8503, Japan

Tel: +81-88-633-7240, Fax: +81-88-633-0178, E-mail: kosahigasino@gmail.com 
technique is widely used because of its safety and ease. Appropriate screw trajectory based on the entry point is important to avoid nerve root injury, vertebral artery injury, or facet joint violation. The entry point used in the Roy-Camille technique is at the center of the articular mass confirmed via direct visualization during surgery [1-3]. Although the Roy-Camille technique has been considered reliable, determining the entry point at a lateral or cephalad angle remains problematic during surgery.

The main causes for problems in determining the entry point are severe degenerative spondylotic changes of the facet joints such as osteophytic spurs of the inferior articular process, particularly those that overlap onto the lateral mass, which cause the center of the lateral mass to be shifted to the caudal level [4]. There is a need for more accurate landmarks to determine the entry point for lateral mass screwing in the Roy-Camille technique.

The vertebral lateral notch on the lateral mass was introduced as a topographic bony landmark for the entry of posterior cervical pedicle screw [5]. Preoperative reconstructed computed tomography (CT) is useful to assess lateral mass anatomy in each patient and for decision-making to avoid surgical complications and poor outcomes [6]. The lateral notch is defined as the lateral shallow concavity of the superior articular process on the coronal plane using three-dimensional CT. During surgery, the lateral notch can be exposed to prepare for the placement of the lateral mass screw.

No studies have reported consistent and reliable landmarks as entry points for lateral mass screwing. The purpose of this study was to investigate whether the vertebral lateral notch of the cervical spine is an effective landmark.

\section{Materials and Methods}

The study involved 33 female patients with cervical spondylotic myelopathy who underwent reconstructed CT scanning of the cervical spine at Tokushima University Hospital between April 2004 and September 2010. The mean age of patients was 62.6 years (range, 37-79 years). All patients subsequently underwent cervical surgery for cervical myelopathy. Cervical vertebrae were preoperatively examined using a multislice scanner (Toshiba Aquilion 16; Toshiba Medical Systems, Tokyo, Japan). Image data were obtained in 1-mm slices, reconstructed using the high-resolution kernel, and transferred onto digital software (Aquarius NET Server; Terarecon Inc., Tokyo, Japan). The software generated three-dimensional (3D) orthogonal (axial, sagittal, and coronal) scans and performed multiplane synchronization.
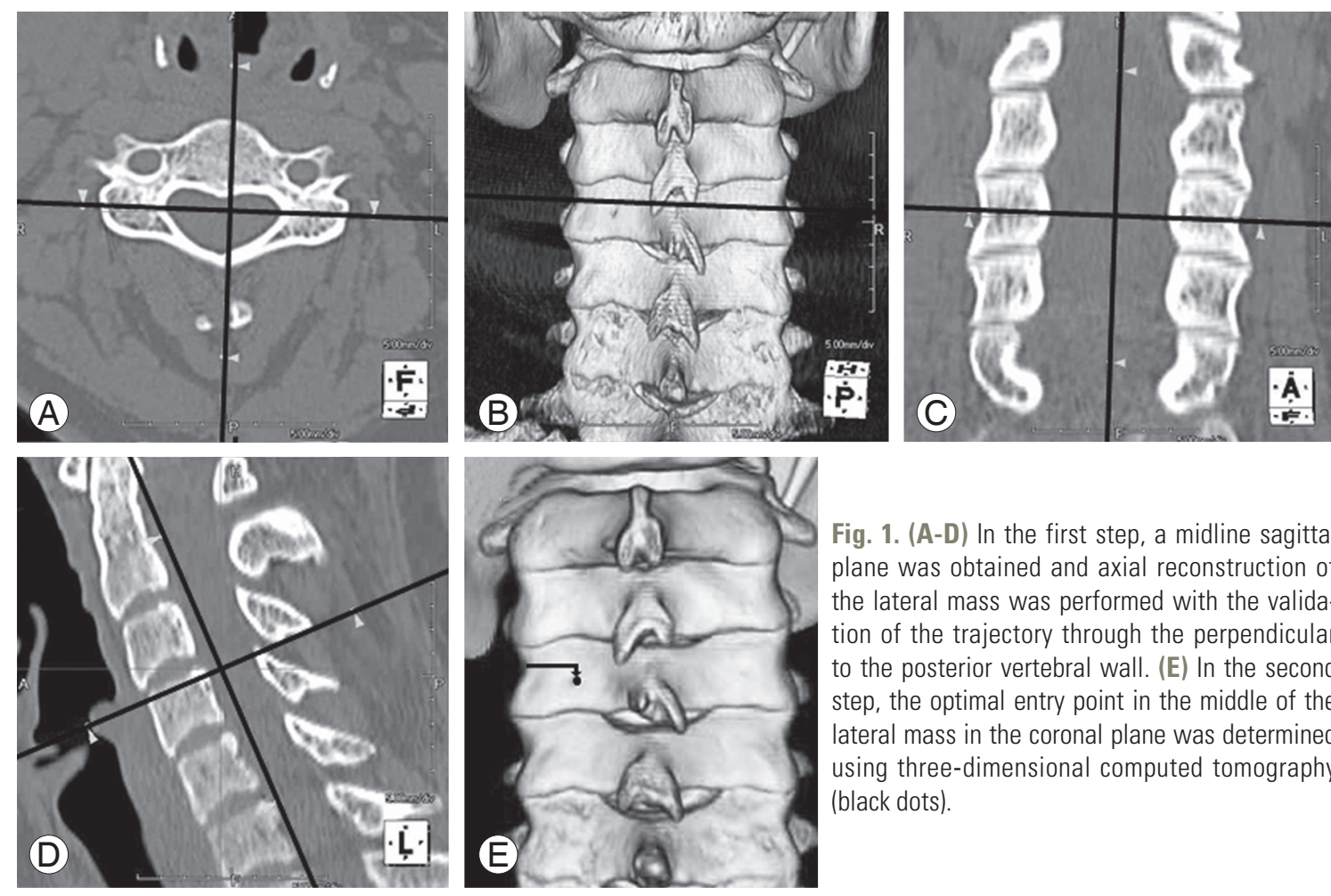

Fig. 1. (A-D) In the first step, a midline sagittal plane was obtained and axial reconstruction of the lateral mass was performed with the validation of the trajectory through the perpendicular to the posterior vertebral wall. (E) In the second step, the optimal entry point in the middle of the lateral mass in the coronal plane was determined using three-dimensional computed tomography (black dots). 
We determined the entry point using the Roy-Camille technique on 3D-CT scans using the procedure mentioned below. Roy-Camille et al. [1] have reported that the entry point must be situated lateral to the valley, on the top of the hill, exactly in the middle of the articular mass.

\section{Scanning protocol}

Our modified Roy-Camille technique was based on 3D orthogonal (axial, sagittal, and coronal) CT scans with mulitplane synchronization. First, a midline sagittal plane was obtained and axial reconstruction of the lateral mass was performed with the validation of the trajectory through the perpendicular to the posterior vertebral wall. Second, the optimal entry point in the middle of the lateral mass in the coronal plane was determined using 3DCT (Fig. 1).

\section{Evaluation of the technique}

To evaluate the effectiveness of the vertebral lateral notch as a landmark for determining the entry point, we measured the distance from the lateral notch to the entry point using both conventional and modified Roy-Camille technique. Measurements were performed by two independent observers via random selection (N.Y. and H.K.). All values were rounded to the nearest millimeter.

\section{Statistical analysis}

Mean values and standard deviations of the medial and inferior distances were compared using analysis of variance and post hoc tests at a 95\% confidence level. All $p$ values were two-sided and considered statistically signifi-

Table 1. Assessment of the vertical entry point, the inferior distance from the vertebral lateral notch to the entry point for each cervical level (left and right sides combined)

\begin{tabular}{lcc} 
Variable & $\begin{array}{c}\text { Roy-Camille } \\
\text { technique }\end{array}$ & $\begin{array}{c}\text { Modified Roy-Camille } \\
\text { technique }\end{array}$ \\
C3 $(\mathrm{mm})$ & $4.12 \pm 1.07$ & $1.27 \pm 1.07^{\text {a) }}$ \\
C4 $(\mathrm{mm})$ & $4.22 \pm 1.00$ & $1.57 \pm 1.15^{\text {a) }}$ \\
C5 $(\mathrm{mm})$ & $4.07 \pm 1.07$ & $2.30 \pm 1.20^{\text {a) }}$ \\
C6 $(\mathrm{mm})$ & $4.40 \pm 1.12$ & $2.34 \pm 1.04^{\text {a) }}$ \\
\hline
\end{tabular}

Values are presented as mean \pm standard deviation.

${ }^{\text {a) } A n a l y s i s ~ o f ~ v a r i a n c e ~ a n d ~ p o s t ~ h o c ~ t e s t s: ~} \alpha=0.05$. cant at $p<0.05$. To evaluate the effect of body height, it was used as a covariate. All statistical analyses were performed using StatView ver. 5.0 (SAS Institute Inc., Cary, NC, USA).

\section{Results}

Distance from the lateral notch to the entry point in the conventional and modified techniques was measured (Tables 1,2$)$. The entry point using the modified technique was significantly superior and medial compared with that using the conventional Roy-Camille technique $(p<0.05)$.

\section{Assessment of the vertical entry point}

Overall, at all levels, the entry points using the conventional Roy-Camille technique were $4.0 \mathrm{~mm}$ below the lateral notch. In contrast, at C3 and C4 levels, the entry point using the modified technique was $1.4 \mathrm{~mm}$ below the lateral notch, and at C5 and C6 levels, it was $2.3 \mathrm{~mm}$ below the lateral notch (Table 1).

\section{Assessment of the horizontal entry point}

At C3 and C4 levels, the entry point using the conventional Roy-Camille technique was $2.3 \mathrm{~mm}$ medial to the lateral notch, and at C5 and C6 levels, it was $3.5 \mathrm{~mm}$ medial to the lateral notch. In contrast, at $\mathrm{C} 3$ and $\mathrm{C} 4$ levels, the entry point using the modified technique was $4.4 \mathrm{~mm}$ medial to the lateral notch, and at C5 and C6 levels, it was $4.9 \mathrm{~mm}$ medial to the lateral notch (Table 2).

These results suggested that our modified Roy-Camille technique was significantly superior and medial compared with the conventional Roy-Camille technique (Fig. 2). The

Table 2. Assessment of the horizontal entry point, the medial distance from the vertebral lateral notch to the entry point for each cervical level (left and right sides combined)

\begin{tabular}{lcc} 
Variable & $\begin{array}{c}\text { Roy-Camille } \\
\text { technique }\end{array}$ & $\begin{array}{c}\text { Modified Roy-Camille } \\
\text { technique }\end{array}$ \\
C3 $(\mathrm{mm})$ & $2.66 \pm 0.81$ & $4.43 \pm 0.87^{\text {a) }}$ \\
C4 (mm) & $2.93 \pm 0.80$ & $4.43 \pm 0.52^{\text {a) }}$ \\
C5 $(\mathrm{mm})$ & $3.45 \pm 0.76$ & $4.74 \pm 0.70^{\text {a) }}$ \\
C6 $(\mathrm{mm})$ & $3.78 \pm 0.83$ & $5.00 \pm 0.74^{\text {a) }}$ \\
\hline
\end{tabular}

Values are presented as mean \pm standard deviation.

a) Analysis of variance and post hoc tests: $\alpha=0.05$. 

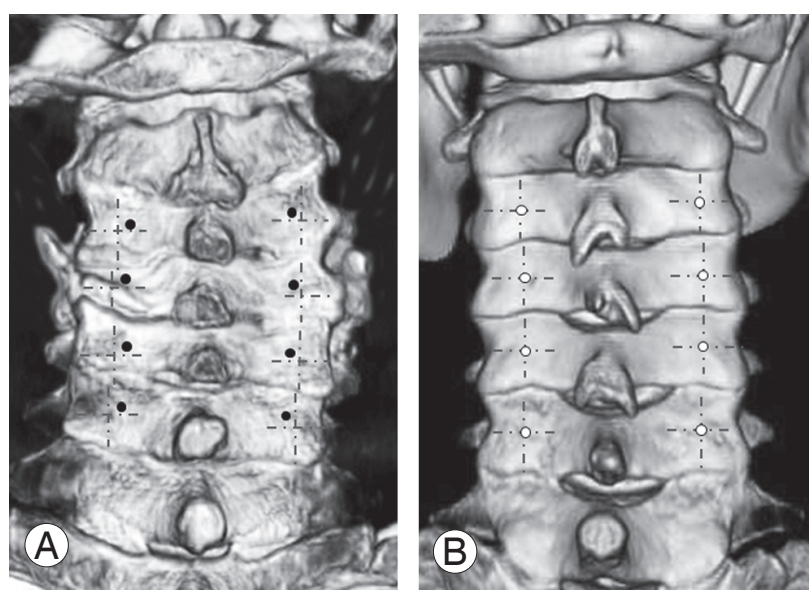

Fig. 2. (A) Entry points in the modified Roy-Camille technique are shown as black dots in a degenerative spondylotic case. (B) Entry points in the conventional Roy-Camille technique are shown as white circles in a nondegenerative spondylotic case. (A) Entry points in our modified Roy-Camille technique were significantly superior and medial compared with those in the conventional Roy-Camille technique.

modified technique would be particularly useful in cases with degenerative spondylotic changes of the facet joints such as osteophytic spurs of the inferior articular process, particularly those that overlap onto the lateral mass, which cause the center of the lateral mass to be shifted to the caudal level.

\section{Discussion}

The main results of this study were as follows: (1) The vertebral lateral notch of the cervical spine was useful as a landmark in determining the entry point for lateral mass screwing and (2) the entry points in the modified RoyCamille technique were significantly superior and medial to the lateral mass than those in the conventional RoyCamille technique.

Anatomic structures at risk during the lateral mass screwing of the subaxial cervical spine are the vertebral artery, nerve root, and facet joint. Our previous study has shown no neurovascular complications in 63 lateral mass screws until the year 2007 [7]. Heller et al. [2] have reported a higher incidence of nerve root injury using the Magerl technique than using the Roy-Camille technique. The main anatomic risk in the Roy-Camille technique is facet joint violation, particularly at the lower part of the cervical spine C5-C6 [2,3,8].

Any landmark should be consistent, its location should be easily found, and it should be reliable. Several stud- ies have reported on the methods used to increase the accuracy of lateral mass screwing, including using the spinous process [9]. Even if posterior structures of the cervical spine showed severe changes such as degenerative spondylosis or were affected after laminectomy [9], the accurate identification of the entry point for lateral mass screwing was found to be feasible using the lateral notch as a landmark. 3D-CT can provide accurate images of the lateral mass. To ensure an accurate screwing, the surgeon can confirm screw trajectory using the position of the landmark relative to the lateral notch based on 3D-CT.

\section{Conclusions}

The vertebral lateral notch of the cervical spine is an effective landmark to determine the entry point for lateral mass screwing. Our modified Roy-Camille technique can be a safe and reliable method for spine surgeons even in the presence of degenerative cervical spondylotic changes.

\section{Conflict of Interest}

No potential conflict of interest relevant to this article was reported.

\section{References}

1. Roy-Camille R, Saillant G, Laville C, Benazet JP. Treatment of lower cervical spinal injuries: C3 to C7. Spine (Phila Pa 1976) 1992;17(10 Suppl):S442-6.

2. Heller JG, Carlson GD, Abitbol JJ, Garfin SR. Anatomic comparison of the Roy-Camille and Magerl techniques for screw placement in the lower cervical spine. Spine (Phila Pa 1976) 1991;16(10 Suppl):S552-7.

3. Barrey C, Mertens P, Jund J, Cotton F, Perrin G. Quantitative anatomic evaluation of cervical lateral mass fixation with a comparison of the Roy-Camille and the Magerl screw techniques. Spine (Phila Pa 1976) 2005;30:E140-7.

4. Xu R, Ebraheim NA, Yeasting R, Wong F, Jackson WT. Anatomy of C7 lateral mass and projection of pedicle axis on its posterior aspect. J Spinal Disord 1995;8:116-20.

5. Karaikovic EE, Kunakornsawat S, Daubs MD, Madsen TW, Gaines RW Jr. Surgical anatomy of the cervi- 
cal pedicles: landmarks for posterior cervical pedicle entrance localization. J Spinal Disord 2000;13:63-72.

6. Higashino K, Sairyo K, Katoh S, Nakano S, Enishi $\mathrm{T}$, Yasui $\mathrm{N}$. The effect of rheumatoid arthritis on the anatomy of the female cervical spine: a radiological study. J Bone Joint Surg Br 2009;91:1058-63.

7. Sairyo K, Sakai T, Higashino K, Tamura T, Katoh S, Yasui N. Cervical and upper thoracic screwing for spinal fusion: strategy for its safe insertion to avoid major complications. Arch Orthop Trauma Surg 2009;129:1447-52.

8. Jonsson H Jr, Rauschning W. Anatomical and morphometric studies in posterior cervical spinal screwplate systems. J Spinal Disord 1994;7:429-38.

9. Stevens QE, Majd ME, Kattner KA, Jones CL, Holt RT. Use of spinous processes to determine the optimal trajectory for placement of lateral mass screws: technical note. J Spinal Disord Tech 2009;22:347-52. 